\title{
CYCLOTOMIC INVARIANTS FOR PRIMES TO ONE MILLION
}

\author{
R. ERNVALL AND T. METSÄNKYLÄ
}

\begin{abstract}
Our recent computation of cyclotomic invariants for primes between 125000 and 150000 was extended to one million. No new phenomena appear.
\end{abstract}

This note is a sequel to our recent report [2] on the computation of certain cyclotomic invariants for primes $p$ between 125000 and 150000 . That work was based on the table of irregular primes supplied by Tanner and Wagstaff (see [4]). Meanwhile, the extension of this table to $p<10^{6}$ by Buhler, Crandall, and Sompolski [1] enabled us to go on with our computations, and we indeed ran the main part of our earlier program in this new range.

The result is that all the previous statements remain valid. To make this precise, we first introduce the necessary notation; for a more thorough discussion of the notions involved, the interested reader is referred to [2] and [5]. For an odd prime $p$ and for $n \geq 0$, let $h_{n}$ and $A_{n}$ denote the class number and $p$-class group, respectively, of the cyclotomic field $K_{n}$ generated by the $p^{n+1}$ th roots of 1 , and let $h_{n}^{-}$and $A_{n}^{-}$stand for their minus-parts. Denote by $r_{p}$ the index of irregularity of $p$, that is, the number of $p$-divisible Bernoulli numbers $B_{t}$ with $t \in I=\{2,4, \ldots, p-3\}$.

Our computations now allow us to state that

$$
A_{n}^{-} \simeq\left(\mathbb{Z} / p^{n+1} \mathbb{Z}\right)^{r_{p}} \quad(n=0,1, \ldots), \quad \operatorname{ord}_{p}\left(h_{0}^{-}\right)=\operatorname{ord}_{p}\left(B_{2} B_{4} \cdots B_{p-3}\right)
$$

for all $p<10^{6}$, where $\operatorname{ord}_{p}(a)$ denotes the exponent of $p$ in the canonical decomposition of $a$.

Note that the computations of [1], together with earlier computations by Wagstaff et al., imply that $A_{n}^{-} \simeq A_{n}$ for these $p$. From this and (1) it follows, among other things, that the $\lambda$-invariant of the $\mathbb{Z}_{p}$-extension $\bigcup_{n=0}^{\infty} K_{n}$ over $K_{0}$ equals $r_{p}$ in this range.

It is worth pointing out that the results in [1] also imply the truth of Fermat's Last Theorem for all prime exponents up to one million.

We now describe briefly the contents of our computations. Let $(p, t)$ be an irregular pair, i.e., a pair with $t \in I$ and $p \mid B_{t}$. As in [2], put

$$
S_{1}=\sum_{0<a<p / 2} a^{t-1} q_{a}, \quad S_{2}=\sum_{0<a<p / 2} a^{t} q_{a}^{2},
$$

Received by the editor April 15, 1991.

1991 Mathematics Subject Classification. Primary 11R18, 11B68, 11R23, 11R29, 11Y40.

Key words and phrases. Cyclotomic fields, Bernoulli numbers, irregular primes, Iwasawa invariants, class numbers, computation. 


$$
S_{3}=\sum_{0<a<p / 2} a^{t-1}, \quad S_{4}=\sum_{0<a<p / 3} a^{t-1}, \quad S_{5}=\sum_{p / 3<a<p / 2} a^{t-2},
$$

where $q_{a}$, the Fermat quotient of $a$, is defined by the conditions $0 \leq q_{a}<p$, $q_{a} \equiv\left(a^{p-1}-1\right) / p(\bmod p)$. Our Criteria $1-4$ proved in [2] imply that $(1)$ holds true provided each irregular pair $(p, t)$ satisfies one of the following two conditions:

(a) both $2^{t}-1$ and $S_{1}$ are nondivisible by $p$ and both $S_{3}$ and $S_{3}-(1-t) p S_{1}$ are nondivisible by $p^{2}$,

(b) the prime $p$ divides $2^{t}-1$ but both $3^{t}-1$ and $S_{2}$ are nondivisible by $p$, and both $3 S_{4}-(1-t) p S_{5}$ and

$$
3 S_{4}-(1-t) p S_{5}+\left(\frac{2}{3}\right)^{t-2} \frac{3^{t}-1}{2^{t-1}-1}(1-t) p S_{2}
$$

are nondivisible by $p^{2}$.

For the connections of (a) and (b) with the divisibility of Bernoulli numbers, see [2].

Checking each irregular pair $(p, t)$ with $1.5 \cdot 10^{5}<p<10^{6}$ for the condition (a) we found that this condition is satisfied by all pairs except $(599479,359568)$. This pair was checked to satisfy (b).

When calculating $S_{3}$ and $S_{4}\left(\bmod p^{2}\right)$ the program verified that these sums vanish modulo $p$. This provides a check for the irregularity of the given pairs $(p, t)$; see Proposition 5 in [2].

As before, the computer we used was a VAX 6340. Because of the extensiveness of the data material, new efforts were made to minimize the running time. While the actual computer program remained unchanged, we slightly rearranged the order of some calculations and enlarged the working set in the virtual machine, reaching in this way a time of about $p / 10^{4}$ seconds for a single irregular pair $(p, t)$.

R. Sompolski informed us that he has established (1) for $p<400000$ by a computation based on an independent method (see [3]).

\section{ACKNOWLEDGMENT}

We thank Professor Joe Buhler and his coauthors for permitting us to use their table.

\section{BIBLIOGRAPHY}

1. J. P. Buhler, R. E. Crandall, and R. Sompolski, Irregular primes to one million, Math. Comp., (to appear).

2. R. Ernvall and T. Metsänkylä, Cyclotomic invariants for primes between 125000 and 150000 , Math. Comp. 56 (1991), 851-858.

3. R. Sompolski, Thesis, Univ. of Illinois at Chicago, 1991.

4. J. W. Tanner and S. S. Wagstaff, Jr., New congruences for the Bernoulli numbers, Math. Comp. 48 (1987), 341-350.

5. L. C. Washington, Introduction to cyclotomic fields, Springer-Verlag, Berlin and New York, 1982. 